\title{
Best Overall Response
}

National Cancer Institute

\section{Source}

National Cancer Institute. Best Overall Response. NCI Thesaurus. Code C94536.

The most clinically favorable response recorded from the start of the study treatment until the end of treatment. 